\title{
Dynamic HDIL Responses While Saline Drilling Mud Invading Reservoirs
}

\author{
Jianhua Zhang \\ Science College, Xi'an Shiyou University, Xi'an, 710065, China \\ e-mail: jhzhang@xsyu.edu.cn
}

Keywords: well logging; high definition induction log; invasion; time; saline mud

\begin{abstract}
A high definition induction log (HDIL) device provides six resistivity responses with various radial detective depths. Its responses were influenced by saline mud filtrate invading into the reservoir dynamically after the formation was opened while drilling. The dynamic invasion process results in the time dependent formation-resistivity profiles, thus the responses of HDIL are time dependent. The measurement results deviate from the true-formation resistivity, so that errors and wrong estimation would be introduced. The effects of saline mud on dynamic responses of HDIL devices were calculated from the dynamic invasion theory for oil bearing reservoir, water zone and oil/water formation. The shallow detective HDIL arrays are influenced by the invasion process strongly. The logging time must be regarded as an important parameter to estimate the true-formation resistivity while evaluating the reservoirs and understanding the dynamic invasion process physically. Well logging was suggested to be performed at the early stage when the formation was opened for less effects of invasion.
\end{abstract}

\section{Introduction}

During petroleum exploration, hydrocarbon saturation is an important parameter for reservoir evaluation. However, it can not be measured directly. It can be estimated from other formation parameter such as formation resistivity.

Induction log is an important method to measure formation resistivity. Traditional dual-induction log tool provides only two apparent resistivities with medium and deep detective depth respectively [1]. Whereas a new array-type induction devices, High-Definition Induction Log (HDIL)[2], can provide more messages about a reservoir. It is a multiarray induction logging tool that measures the formation resistivity simultaneously with 7 arrays at 8 operating frequencies and it can provide six depths of investigation, $10,20,30,60,90$, and $120 \mathrm{inch}(25,51,76,152,229$, and 305cm) respectively.

However, the measurement of formation resistivity is influenced by invasion process. During drilling, mud filtrate invades into permeable and porous formations, thus invaded zone was formed. Saline mud has the properties of low viscosity, high conductivity and good rheology. It is also helpful to avoid the sidewall shrink and collapse. Its thermal stability is good for deep well drilling. However, it has high conductivity and easy leads the logging responses less than the true-formation resistivity. Thus, errors and wrong evaluation were introduced [3].

In addition, most of the investigations for the invasion process of saline drilling-mud filtrate were based on a conventional step-invasion model [4] that presumes the resistivity varies sharply at the boundary of invaded zone and undisturbed formation. However, a realistic invasion process is much complex. At the beginning of drilling a formation, the rate of invasion is fast. With the lapse of time, mud cake is built at the wall of borehole; then the rate of invasion becomes slowly. Hence, the formation- and fluid-related parameters do not vary in a step invasion style and the invasion process is related to time. The dynamic invasion process and its effect on resistivity logging tools have been investigated in computer simulation and field applications [5-7]. The dynamic invasion process of saline mud filtrate causes strong effect on HDIL devices, so that its measurements will also be time dependent. 
The theoretical model of dynamic invasion, which based on fluid flowing theory, was used to calculate the invasion profiles of formation parameters. Then the dynamic responses of a HDIL device can be calculated. The departure between the measurement data and formation true value were obtained for saline mud filtrate. The present results can give a reasonable interpretation for the measurement deviations from the true values. The time-dependent invasion profiles and the behaviors of dynamic HDIL responses for oil bearing reservoir, water zone and oil/water formation were calculated respectively. They can provide more information for log analysts to evaluate the reservoirs when saline mud was used.

\section{Dynamic Invasion}

After the formation was opened, drilling mud filtrate was driven by the pressure difference between the borehole and formation; Then the mud filtrate invaded into the reservoir. The present formation model included the borehole and a thick bed without shoulders. The mud filtrate invades into a formation radially and displaces the native fluids in porous volumes. The displacement between filtrate and hydrocarbon is immiscible.

The invasion profiles will change at various time stages, and they can be calculated from fluid flow equations. The invasion process is related to formation porosity $\varphi$, native-water salinity $C_{w}$ and mudfiltrate salinity $C_{m f}$, absolute permeability $k$, relative permeability $k_{r i}$, original saturation $S_{i}$, formation pressure $P_{i}$, viscosity $\mu_{i}$ in formation, the flow capacity $q_{i}$, here the subscript $i=o, w$ means oil phase and water phase respectively. While the mud filtrate invading into the formation, both saturation and pressure are functions of time $t$ and radial position $r$. The water-phase saturation, oil-phase saturation, water-phase pressure and oil-phase pressure can be solved from flow equations [8]:

$$
\frac{1}{r} \frac{\partial}{\partial t}\left(\frac{r k k_{r i}}{\mu_{i}} \frac{\partial P_{i}}{\partial t}\right)+q_{o}=\frac{\partial\left(\varphi P_{i} S_{i}\right)}{\partial t} \quad i=o, w
$$

In addition, the salinity of mud filtrate, $C_{m f}$, is usually different from that of formation water, $C_{w}$. The mixing of mud filtrate with native water causes the variation of salinity distribution with time during invasion. The variation of water salinity is generated from following dispersion equation:

$$
\frac{1}{r} \frac{\partial}{\partial r}\left(\frac{r k k_{r w} C_{w}}{\mu_{w}} \frac{\partial P_{w}}{\partial r}\right)+q_{w} C_{m f}=\frac{\partial\left(\varphi S_{w} C_{m f}\right)}{\partial t}
$$

After $\mathrm{C}_{w}$ was obtained, the formation water resistivity, $R_{w}$, can be solved from the petrophysics theory [9]:

$$
R_{w}=\left(0.0123+\frac{3647.5}{C_{w^{\prime}}^{0.955}}\right) \cdot \frac{82}{39+1.8 T}
$$

The formation resistivity can be calculated from Archie’s equation [10]:

$$
R_{f}=\frac{a b R_{w}}{S_{w}^{n} \varphi^{m}}
$$

Where, both $a$ and $b$ are constants, $m$ is the cementation factor, and $n$ is the saturation exponent.

Thus, (1) (4) were the main equations of dynamic invasion process. They determine the invasion profiles of saturation, pressure, salinity, formation resistivity and formation-water resistivity

\section{Dynamic Responses of HDIL Devices}

The invasion profile of formation resistivity at time $t$ determines the apparent resistivity of HDIL devices. A HDIL device is composed by seven three-coil arrays at 8 operating frequencies (10, 30, 50, 
70, 90, 110, 130 and $150 \mathrm{kHz}$, respectively). Its apparent resistivity $R_{a}$ can be obtained from following equation:

$$
\frac{1}{R_{a}}=\operatorname{Re} \sum_{j=1}^{n} \sum_{k=1}^{m} \frac{w_{k j}}{R_{a}^{(j)}\left(z-z_{k j}\right)}
$$

Where $R_{a}^{(j)}$ is apparent resistivity of $j$-th three-coil array. $w_{j k}$ is weight factor. $n$ and $m$ are the number of receive and transmit coils respectively. The $z$ axis is along the direction of device length. Re means the real parts of the complex function.

The dynamic HDIL responses can be yielded from (1) (5) for six detective depths: 10, 20, 30, 60, 90, and 120inch $(25,51,76,152,229$, and $305 \mathrm{~cm})$ respectively. The six measured resistivities are usually marked as HDIL10, HDIL20, HDIL30, HDIL60, HDIL90 and HDIL120.

\section{Effects of Saline Mud Invasion on HDIL}

The dynamic invasion effects of saline mud on a HDIL device were calculated using present model for oil bearing reservoir, water zone and oil/water formation respectively. The necessary input dada are: initial formation-water salinity $C_{w}=20000 \mathrm{mg} / \mathrm{L}$, mud salinity $C_{m f}=150000 \mathrm{mg} / \mathrm{L}$, pressures of borehole and original reservoir were $40 \mathrm{MPa}$ and $35 \mathrm{MPa}$ respectively; other main input data were: $\varphi=0.15$, $k=0.02 \mu \mathrm{m}^{2}, T=105^{\circ} \mathrm{C}, m=2, n=2$, and $a=b=1$ in Archie's equation.

\section{An oil bearing reservoir}

The present algorithm was first used to solve the case of saline mud filtrate invading into an oil bearing reservoir dynamically. The initial water saturation $S_{w}$ was set to be 0.1 , thus the oil saturation was $90 \%$ to address an oil bearing reservoir.

Fig.1a illustrated the radial profiles of formation resistivity obtained from (1) (4). The horizontal axis denoted the distance from borehole. The vertical axis was the values of formation resistivity. At the beginning of mud invasion, $t=0$, the resistivity of borehole was low due to the high salinity mud and the initial formation resistivity was high for the reason of an oil bearing reservoir. The sharp boundary was the wall of the borehole. After the formation was opened, the saline mud filtrate moved into the reservoir gradually, the profiles of formation resistivity changed with time. They were drawn in Fig.1a and marked with 3, 6, 14 and 23day, respectively, to illustrate the invasion profiles at each time stage of the invasion process. These curves show that the invasion occurred and the invasion profile moved to the reservoir with time. After 20 days of the bit penetrating, the invasion depth reached 20 meters, as shown in fig.1a. The resistivity does not vary sharply at the boundary described as step-invasion model [3].

The variation of resistivity profiles with time makes a notable impact on HDIL responses. It is easy to understand that the resistivity logging measurement departs from the actual formation parameter and it is not a constant, but variable data depending on the time, as shown in Fig.1b. In Fig.1b, the horizontal axis denoted the invasion time and the vertical axis was the apparent resistivity of HDIL measurement calculated by (5).

For an oil-bearing reservoir with less water saturation $S_{w}$. Since an oil zone contains little movable water, the high-resistivity hydrocarbon was displaced by saline mud filtrate that had high salinity and low resistivity. The resistivity in the zone near the borehole was low. It affects the shallow detective arrays such as HDIL 10 and HDIL 20 strongly; hence they have low responses. During the whole invasion period, the deeper the detective depth is, the greater the apparent resistivity is. Thus, HDIL120 > HDIL90 > HDIL60 > HDIL30 > HDIL20 > HDIL10, as shown in Fig.1b. It is obvious that when the low-resistivity mud filtrate reached the detective range of a HDIL device, the measurement data were less than that of true formation resistivity.

For example, at 10 days of the invasion occurrence, the HDIL measurement gave apparent

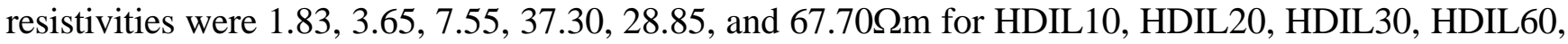


HDIL90 and HDIL120 respectively. All of them were less than the true-formation resistivity $R_{t}=$ $474.87 \Omega \mathrm{m}$. The effect of saline mud filtrate on HDIL measurement was obviously. Only the well was logged within 3 days of invasion occurrence, the apparent resistivity of deep detective HDIL120 was close to the true value of reservoir.

\section{A water bed}

If the reservoir is filled by movable water, it has high formation-water saturation. In the present simulation, the initial water saturation $S_{w}$ was set to be 0.9 for a water bed. The native water with low salinity, thus high resistivity, was replaced by mud filtrate that has high salinity, thus low resistivity.

Figure 2a illustrated the radial profile of formation resistivity at various time stages $(3,6,14$, and 23 days respectively) after the bed was reached. The initial water saturation $S_{w}$ was equal to 0.9 for a water bed for present calculation. Other input data were same as Fig.1.
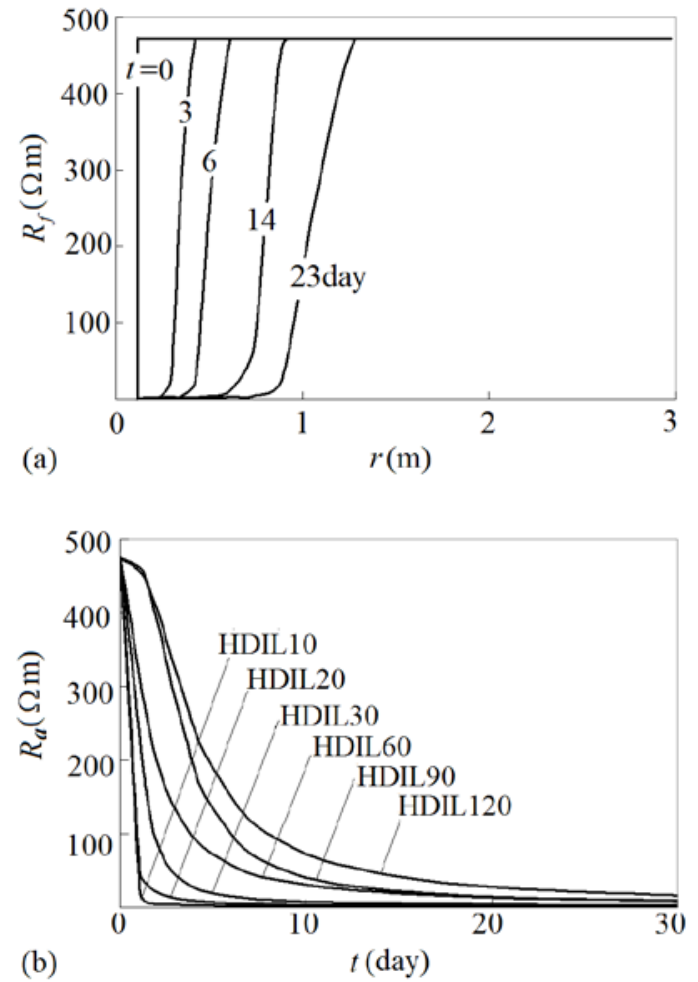

Figure 1. An oil bearing reservoir: (a) formation-resistivity invasion profiles; (b) dynamic HDIL responses

Figure $2 \mathrm{~b}$ draws the resistivity responses of HDIL device corresponding to the dynamic radial invasion profiles in Fig.2a. For the same reason as Fig.1, the low-resistivity zone close to the wellbore resulted in the less readings of shallow detective HDIL array. Hence, the present computation generated the result HDIL120 > HDIL90 > HDIL60 > HDIL30 > HDIL20 > HDIL10, as shown in Fig.2b. The range in magnitude for the present simulation results of HDIL apparent resistivities is the same as saline mud filtrate invading into an oil bearing reservoir, as shown in Fig.1b.

When saline mud filtrate invading a water zone, the deep detective array, HDIL90 or HDIL120, can still address the characters of the water reservoir after the reservoir was immersed for one month; whereas the readings of shallow array such as HDIL10 and HDIL20 generate obvious errors, as shown in Fig.2b.

An oil-water zone

In the present study, the initial water saturation $S_{w}$ was equal to 0.5 to address a typical oil-water bed. Other input parameters during the present simulation were as same as in Fig.1 and Fig.2. The four curves in Fig.3a illustrated the radial profiles of formation resistivity at various time stages $(3,6,14$, and 23 days respectively) after the bed was reached. 

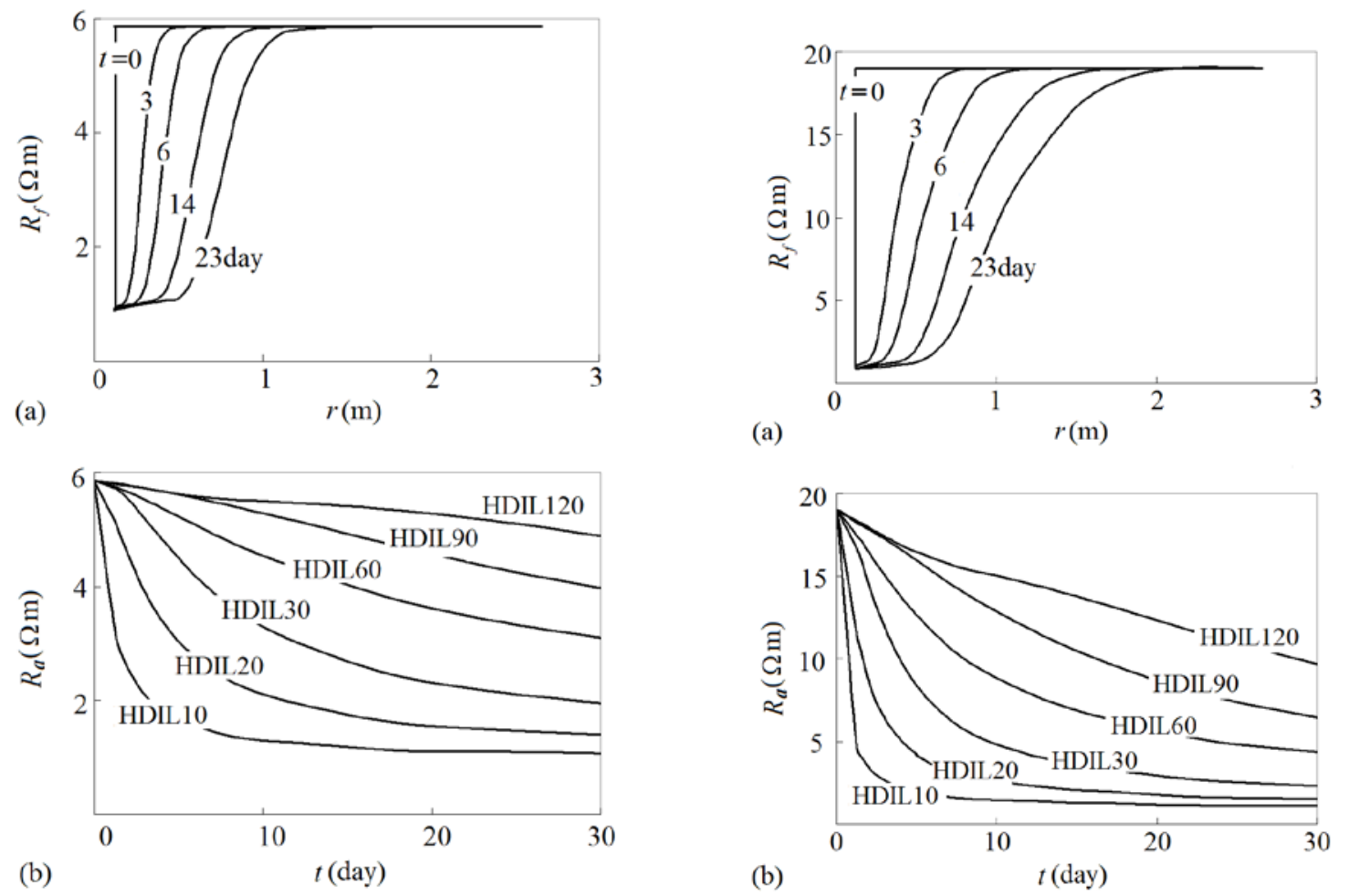

Figure 2. A water bed: (a) formation-resistivity invasion profiles; Figure 3. An oil-water bed: (a) formation-resistivity invasion profiles;

(b) dynamic HDIL responses.

(b) dynamic HDIL responses.

When saline mud filtrate invading a reservoir that contains both hydrocarbon and water equivalently, the resistivity in the invading zone was lower than the value in undisturbed formation, as shown in Fig.3a. Therefore, the low-resistivity invasion zone affected the readings of sallow detective HDIL array heavily. At the same measurement time, the apparent resistivity of HDIL tool decreased in the sequence of HDIL120, HDIL90, HDIL60, HDIL30, HDIL20, and HDIL10. The deep detective HDIL arrays generate less measurement errors than the shallow array because they get less influence by the invasion of mud filtrate.

\section{Conclusions}

The process of saline mud filtrate invading into a reservoir has strong impact on the measurements of high-definition induction log. It may causes serious errors and lead to the measurement results deviates from the true-formation resistivity obviously. Thus, mistaking estimation would be introduced.

The present dynamic responses of HDIL tools can not only provide the dynamic invasion profiles, but also generate the time-dependant apparent resistivity responses. It is a reasonable algorithm to calculate the dynamic invasion process and logging responses.

When saline drilling mud was used, the low-resistivity mud filtrate invading into a reservoir. No matter an oil-bearing reservoir, water bed, or an oil-water zone, the resistivity near the borehole was low. Thus, the shallow detective HDIL arrays give less apparent resistivities; whereas the deep detective HDIL arrays cause great resistivity responses that approach the true-formation resistivity much more.

The present algorithm provides a better understanding of the basics of dynamic invasion process. It can also be used to eliminate the influence of saline mud on HDIL measurement by history matching $[5,6]$ or inversion technique [11], so that the true-formation resistivity and initial water saturation would be determined. 


\section{References}

[1] J. Tittman, “Geophysical Well Logging”, Orlando:Academic Press Inc., 1986, pp.62-89.

[2] T. D. Barger and R. A. Rosthal, "Using a multiarray induction tool to achieve high-resolution logs with minimum environmental effects", the 66th Annual Techniqucal Conference and Exhibition of the Society of Petroleum Engineers, Dallas, U.S., Paper SPE 22725, 1991

[3] V. Leroux and T. Dahlin, “Time-lapse resistivity investigations for imaging saltwater transport”, Environ Geol. Vol.49, pp347-358, 2006

[4] Elton Head, David Allen and Leif Colsdon, “Quantitative invasion description”, SPWLA 33rd Annual Logging Symposium, ppB1-B21, June 14-17, 1992

[5] D. P. Tobola and S. A. Holditch, "Determination of reservoir permeability from repeated induction logging”, SPE Formation Evaluation, March 1991, pp20-27, 1991

[6] J. H. Zhang, Q. Hu and Z. H. Liu, "Estimation of true formation resistivity and water saturation with a time-lapse induction logging method”, The Log Analyst, Vol.40(2), pp38-148, 1999

[7] J. M. Sun, S.Y. Zheng and J. H. Ma, “A new method for connate water saturation calculation using time-lapse logging data”, J. Pet. Sci. Eng., Vol.50(3-4), pp204-210, 2006

[8] G. W. Thomas, "Principles of Hydrocarbon Reservoir Simulation”, Boston: International Human Resources Development Corporation, p112, 1982

[9] Dresser Atlas Inc., 1982, “Well Logging and Interpretation Techniques”, Dresser Industries, USA, Chap. 3.

[10] G. E. Archie, “The electrical resistivity log as an aid in determining some reservoir characteristics”, Trans. AIME, vol.146, pp54-62, 1950

[11] L. Lin, A. Abubakar, H. Tarek, "Inversion of induction logging data constrained by fluid-flow simulator”, Society of Petroleum Engineers - SPE/IATMI Asia Pacific Oil and Gas Conference and Exhibition 2009, APOGCE 09, vol.2, pp. 664-668, 2009 